\title{
Gene prediction of aging-related diseases based on DNN and Mashup
}

\author{
Junhua Ye, Shunfang Wang ${ }^{*}$, Xin Yang and Xianjun Tang
}

\author{
${ }^{*}$ Correspondence: \\ sfwang_66@ynu.edu.cn \\ School of Information \\ Science and Engineering, \\ Yunnan University, \\ KunMing 650000, China
}

\begin{abstract}
Background: At present, the bioinformatics research on the relationship between aging-related diseases and genes is mainly through the establishment of a machine learning multi-label model to classify each gene. Most of the existing methods for predicting pathogenic genes mainly rely on specific types of gene features, or directly encode multiple features with different dimensions, use the same encoder to concatenate and predict the final results, which will be subject to many limitations in the applicability of the algorithm. Possible shortcomings of the above include: incomplete coverage of gene features by a single type of biomics data, overfitting of small dimensional datasets by a single encoder, or underfitting of larger dimensional datasets.

Methods: We use the known gene disease association data and gene descriptors, such as gene ontology terms (GO), protein interaction data (PPI), PathDIP, Kyoto Encyclopedia of genes and genomes Genes (KEGG), etc, as input for deep learning to predict the association between genes and diseases. Our innovation is to use Mashup algorithm to reduce the dimensionality of PPI, GO and other large biological networks, and add new pathway data in KEGG database, and then combine a variety of biological information sources through modular Deep Neural Network (DNN) to predict the genes related to aging diseases.

Result and conclusion: The results show that our algorithm is more effective than the standard neural network algorithm (the Area Under the ROC curve from 0.8795 to 0.9153), gradient enhanced tree classifier and logistic regression classifier. In this paper, we firstly use DNN to learn the similar genes associated with the known diseases from the complex multi-dimensional feature space, and then provide the evidence that the assumed genes are associated with a certain disease.
\end{abstract}

Keywords: Gene prediction, Gene ontology, Mashup, Deep neural networks, Proteinprotein interaction

\section{Background}

With the increase of aging population, countries all over the world invest more and more in the diseases of the elderly. This also caused more researchers to work to slow down the huge medical costs and social burden caused by human aging. Statistics show that the disease spectrum of human beings is changing from infectious diseases to chronic non infectious diseases such as hypertension, heart disease, stroke and cancer.

(c) The Author(s), 2021. Open Access This article is licensed under a Creative Commons Attribution 4.0 International License, which permits use, sharing, adaptation, distribution and reproduction in any medium or format, as long as you give appropriate credit to the original author(s) and the source, provide a link to the Creative Commons licence, and indicate if changes were made. The images or other third party material in this article are included in the article's Creative Commons licence, unless indicated otherwise in a credit line to the material. If material is not included in the article's Creative Commons licence and your intended use is not permitted by statutory regulation or exceeds the permitted use, you will need to obtain permission directly from the copyright holder. To view a copy of this licence, visit http:// creativecommons.org/licenses/by/4.0/. The Creative Commons Public Domain Dedication waiver (http://creativecommons.org/publi cdomain/zero/1.0/) applies to the data made available in this article, unless otherwise stated in a credit line to the data. 
One of the main problems in the study of human aging is that it is much more difficult to carry out experiments due to obvious ethical reasons and long experimental time, so animal models with shorter life are usually preferred. This problem also creates the opportunity to deploy bioinformatics methods to study human aging. There is a large amount of data on this subject in the publicly available gene or protein database, but such data has not been fully developed [1,2]. The purpose of this work is to analyze the human genes related to aging by combining various kinds of biological data and using deep learning data mining method. After we find the genes related to aging, we can use drugs to repair specific genes to delay human aging and treat human age-related diseases more effectively, which can alleviate the growing pressure of aging society [3]. Therefore, it is particularly important to propose a more effective multi label learning algorithm to reduce the workload of experimental verification in the field of biology $[4,5]$.

There are three contributions of this paper: (1) Using Mashup algorithm to reduce the dimensionality of biological network, and applying the processed data to the prediction of aging disease-related genes [6]; (2) Proposing a new DNN model; (3) According to the prediction of our algorithm, some negative genes close to known positive genes are recommended for further study for each disease. Our proposed architecture is called MDL (Mashup and Deep Learning), which integrates multiple data sources to obtain the prediction of the final model. We compare MDL with existing deep learning architecture [7], gradient boosting tree (GBT) [8]classifier (lightGBM Implementation) and traditional logistic regression (LR) classifier in terms of prediction ability [9].

The rest of this paper is organized as follows: Firstly, describes the construction principle of MDL and the method of compiling data. And then reports our experimental results, including the statistical analysis of the prediction performance of MDL, DNN, GBT and LR classifiers. The priority of disease genes is also the focus of our task, someone proposed Hetesim's method in 2017 [10]. Especially, in the end of this part, a number of promising genes for further analysis are listed based on our method. Finally, in the discussion part, we use hypothesis testing to verify the advantages of the recommended gene compared with other genes. Then, according to the rules of statistical genes, the possibility of further prediction of aging related genes is prospected.

\section{Methods}

\section{Overall structure}

In this paper, we use several types of features (or datasets), as shown in Fig. 1. The KEGG dataset, consisting of gene pathway information from the Kyoto Encyclopedia of Genes and Genomes, was used as described in Fabris and Freitas [11]. We obtained proteinprotein interaction (PPI) features representing the human protein interaction network extracted from the STRING database v9.1 [12] and the Munich Information Center for Protein Sequences (MIPS). The Gene Ontology (GO) features were obtained by downloading data from the GO database [13]. Our method uses the Mashup algorithm [6] and the modular Deep Neural Network (DNN) algorithm proposed in Fabris et al. [7], as summarized in Fig. 1. Firstly, we used the Mashup algorithm to embed the GO terms and PPI features into an 800-dimensional feature vector, generating the Mashup dataset. Second, we use the modular DNN algorithm to learn a model in two phases. In the first phase we train 4 neural networks, denoted as "Encoder 1" in Fig. 1, each trained with 


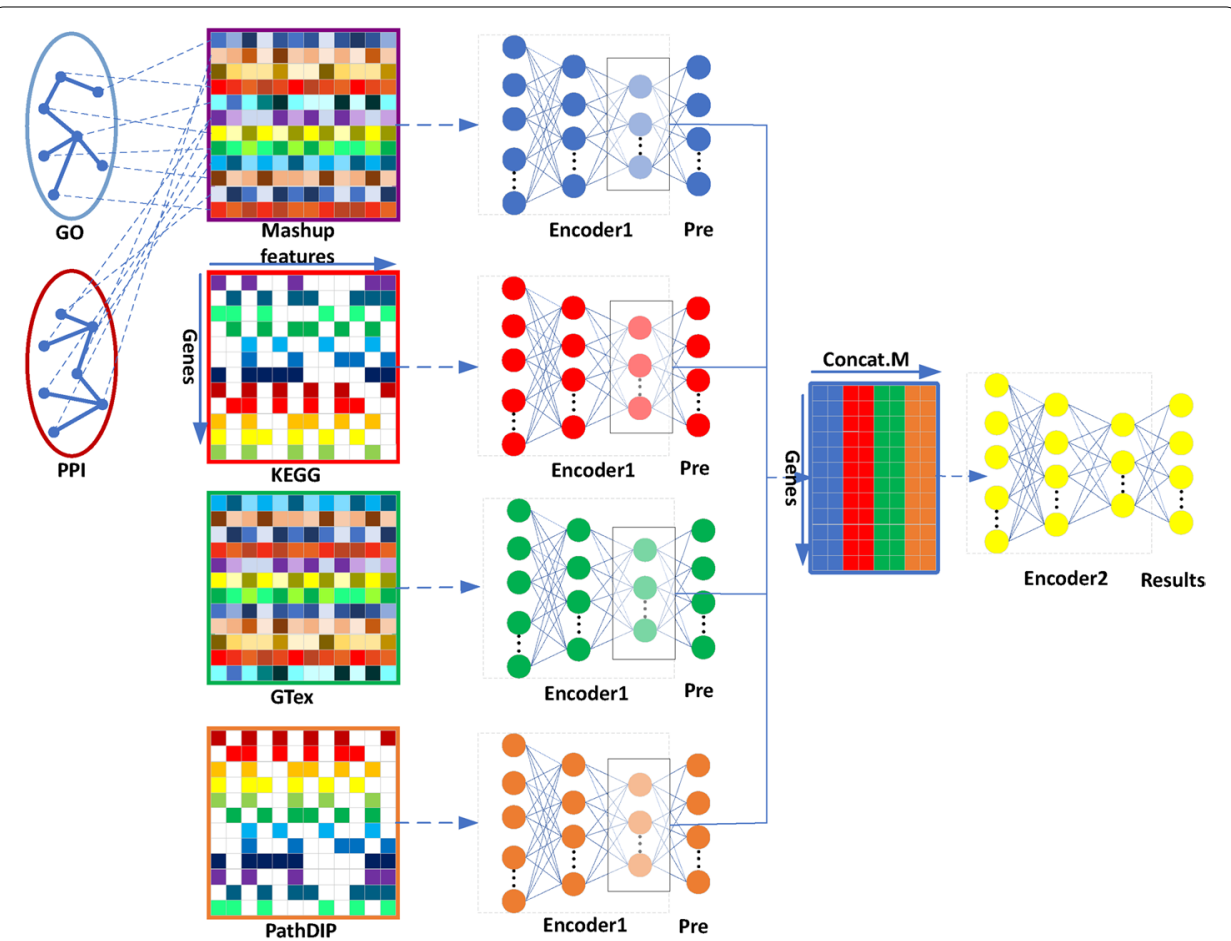

Fig. 1 Algorithm frameworks. The vector of binary features consisted of 4 main parts (PPI, GO, KEGG, PathDIP), each part of which was equivalent to one of the data sources. The information for each data source was a boolean value, and if any gene contained this value, it scored 1, and otherwise, it scored 0 . The remaining part (Mashup, GTEX) is non-BOOL value

a different type of feature (dataset) - namely the Mashup, KEGG, GTEx and PathDIP. Each Encoder 1 is used to reduce the dimensionality of its corresponding dataset. In the second phase, a neural network (denoted as "Encoder 2" in Fig. 1) is trained with a concatenation of the outputs of all trained Encoder 1 neural networks, generating a final predictive model. The specific model is as Fig. 1.

\section{Mashup}

The high-dimensional nature of biological networks and the incompleteness of original networks bring great challenges to the adaptability of machine learning algorithms [14]. Specifically, first, the conflict between the huge amount of computation and the insufficient computation of most current computing devices. Second, the dimensions of multiple biological networks are not of the same order or magnitude, which makes it difficult to integrate multiple biological networks and predict gene function. Therefore, we need an algorithm to merge high-dimensional networks and reduce them into low dimensional data that is basically consistent with other smaller networks. On condition that, we can use an unified deep learning model to learn and predict multiple biological networks, and Mashup is such an excellent algorithm, which can well solve the above challenging problems efficiently.

Mashup algorithm is a network structure analysis tool based on random walk with restart (RWR). RWR is to start random walk again in each step of expansion with a 
certain probability, so as to identify the important nodes in the local and global topology of the network. The RWR from node i is defined as Eqs. $(1,2,3)$ :

$$
\begin{aligned}
& s_{i}^{t+1}=\left(1-p_{r}\right) B s_{i}^{t}+p_{r} e_{i}, \\
& B_{i j}=\frac{A_{i j}}{\sum_{i} A_{i j}}, \\
& e_{i}(j)=\left\{\begin{array}{l}
1, i=j \\
0, i \neq j
\end{array}\right.
\end{aligned}
$$

where $s_{i}^{t}$ is a n-dimensional distribution vector, which holds the probability of reaching each node from $i$ after t-step random walk; $p_{r}$ is the probability of restart; $e_{i}$ is a n-dimensional distribution vector. Each $B_{i j}$ of $B$ represents the probability of a transition from node $i$ to node $j$; $A$ represents the n-dimensional weighted adjacency matrix of the original biological network containing $n$ genes or proteins.

Due to the high dimensionality and incompleteness of biological network, there is a lot of noise in the node diffusion state obtained by restart random walk algorithm. In order to reduce the noise and dimensionality of data, each diffusion state is approximated by a polynomial logic model based on a lower dimensionality latent vector of nodes to generate a much smaller dimensionality than the original feature network.

When there are multiple networks, the above steps are firstly carried out for each network respectively, then the same weights used fuse each network into a final one. The final generation network is a 800 dimensional vector, which is $95 \%$ lower than the original 15,000 dimensional protein interaction network and gene ontology network. The calculation of data analysis is greatly reduced.

\section{Deep neural network model}

With the continuous improvement of computer computing resources, deep learning has also shown its great development potential in various directions in bioinformatics [15, 16]. For example, Daniel et al. [17], Kulmanov et al. [18] used CNN and LSTM to predict nonprotein coding DNA sequences function, Skwark et al. [19] used deep learning network to predict protein structure, Gupta et al. [20] used deep architecture to learn the structure in gene expression data and applied it to gene clustering. It is believed that in the continuous development of deep learning, the prediction of gene expression can be further improved.

In general, the algorithm proposed in this paper has two stages, one is used to encode multiple biological data sources in the early stage, and to detect the coding effect, the other is used to train multiple biological data after serial coding, and give our prediction results. Because of the differences of data sources and data dimensionality, we propose two different deep learning models, Encoder1 and Encoder2.

In the model Encoder1, we use a four-layer fully connected neural network in each initial biological network, whose neurons in each layer are 64, 32, 32 and 16, respectively, and, the output is set to multi-label categories of 27 diseases. The Relu activation function is used in the four hidden layers to ensure that the output is a nonlinear 
combination of inputs, and add a bias neuron to improve the fitting ability of neurons. In the final output layer, we set tanh as the activation function. The combined training of Tanh and Relu activation functions can significantly improve the overall performance of the model [21]. In order to reduce the amount of parameters needed to be calculated in the training model, the learning rate is set to 0.001 , and the neuron dropout rate of each layer to 0.5 . Dropout can prevent over fitting by randomly sets input units to 0 with a frequency of rate at each step during training time. At the end of the training, we keep the last full connection layer data generated by each data set after model training, and then proceed to the next step. In this step of the model Encoder1, the prediction effect of each data set on the disease to be predicted can be initially seen. Although the prediction effect is not the final effect, it can help us measure the embedding effect of this model on each data set. The parameters needed to be trained for each data set are as follows: Para $1=n \times(64+1)+64 \times(32+1)+32 \times(32+1)+32 \times(16+1)+16 \times 27$, where $n$ is the dimensionality of each dataset.

In the final prediction model Encoder2, we use a deep neural network with three hidden layers. The neurons in each layer are 32, 24 and 16, respectively. Bias neurons are added to each layer. As mentioned above, the output is still a multi label vector of length 27. In the activation function, we refer to the data embedding model, using Relu in the hidden layer and tanh in the final output layer. The data we need to input is the data generated by each data set reserved in the previous model, which is connected in series, and then the model is trained. The training parameters of the model are as follows: Para $2=16 \times 4 \times(32+1)+32 \times(24+1)+24 \times(16+1)+16 \times 27=3,436,992$. Table 1 lists the hyperparameters of the deep learning model of Encoder1 and Encoder2.

\section{Results}

\section{Comparison of MDL with other methods}

In this section, we compare our traditional machine learning and deep learning algorithm with several other baseline algorithms, including: (1) existing DNN algorithm, including using a single feature type and concatenating all data sets for training; (2) gradient boosting tree algorithm; (3) logistic regression classifier (LR), using L2 regularization and other default parameters; (4) In order to verify the effectiveness of the Mashup algorithm, we compare the predictive performance of the DNN algorithm using an Encoder 1 module where Mashup was used to process the GO and PPI features together against the DNN algorithm without using Mashup (i.e. using a separate Encoder 1 module for each of the GO and PPI datasets). (5) Compared with the existing deep learning algorithm, the fitting degree of each module and the final prediction effect are compared. (6) The main idea of the Naive classifier is to think that the more research on a gene, the more likely it is to be related to disease. For the GO, PPI and PathDIP datasets, the degree of research is defined as the number of terms. If this gene does not

Table 1 Learning hyperparameters for each network model

\begin{tabular}{lllll}
\hline Models & Batch-size & Optimizer & Learning-rate & Epoch \\
\hline Encoder1 & 2048 & Adam & 0.0001 & 150 \\
Encoder2 & 3072 & Adam & 0.0001 & 150 \\
\hline
\end{tabular}


exist in the data set, it is considered that its research level is 0 . For data sets with nonBOOL values such as GTEx, the degree of genetic research that exists is set to 1 , and the non-existent is set to 0 . Finally, the research level of each gene in the 4 data sets is added together to generate a Naive classifier containing 4 types of feature information to identify whether our algorithm is simply assigning positive classification labels to genes with higher research levels.

Table 2 shows the information of each feature set. Unknown proportion refers to the proportion of the feature set that is not included in the label set. (e.g. proportion of genes without any PPI values, for the PPI feature.) Concat. M is the combination of PathDIP+GTEx+Mashup+KEGG, and Concat. P\&G is the combination of GO + PPI + GTEx + KEGG + PathDIP. It should be noted that the Concat. P\&G row of DNN column and naive column data in the Tables 3 and 4 does not contain KEGG and Mashup data.

Finally, we use the area enclosed by the ROC curve under 10-folds cross validation. The predictive accuracy measure (AUC value in this case) is calculated as the average of the measure in the test set of 10 experiments. Therefore, each data instance is used once in the test set and nine times in the training set. AUC is the area under the ROC curve and surrounded by the coordinate axis, while the abscissa of the ROC curve is the false

Table 2 Data information of each feature set

\begin{tabular}{lllllllll}
\hline Datasets & GO & PPI & PathDIP & GTEx & Mashup & KEGG & Concat. M & Concat. P\&G \\
\hline Unknown proportion & $4 \%$ & $19.4 \%$ & $16.8 \%$ & $4.1 \%$ & $5.3 \%$ & $3.7 \%$ & 0 & 0 \\
Data dimension & 13,615 & 13,887 & 4790 & 84 & 800 & 318 & 5992 & 32,694 \\
Sample size & 18,418 & 15,469 & 15,956 & 18,597 & 15,709 & 7040 & 19,188 & 19,188 \\
\hline
\end{tabular}

Table 3 AUROC values obtained by 10-fold cross-validation in datasets with unknown genes

\begin{tabular}{llllll}
\hline Algorithms/datasets & Encoder1 & GBT & LR & DNN & Naive \\
\hline PPI & 0.7451 & 0.6585 & 0.6844 & 0.6381 & $\mathbf{0 . 7 9 9 5}$ \\
GO & 0.8326 & 0.8520 & 0.7900 & $\mathbf{0 . 8 4 9 8}$ & 0.6080 \\
GTEX & $\mathbf{0 . 8 1 6 5}$ & 0.7156 & 0.7173 & 0.7535 & 0.5062 \\
pathDIP & $\mathbf{0 . 8 4 5 7}$ & 0.8392 & 0.7600 & 0.7507 & 0.7817 \\
Mashup & $\mathbf{0 . 8 6 3 7}$ & NA & NA & NA & NA \\
KEGG & $\mathbf{0 . 8 3 2 2}$ & NA & NA & NA & NA \\
\hline
\end{tabular}

Table 4 AUROC values obtained by 10-fold cross-validation in datasets without unknown genes

\begin{tabular}{llllll}
\hline Algorithms/datasets & Encoder1 & GBT & LR & DNN & Encoder2 \\
\hline PPI & $\mathbf{0 . 7 4 6 4}$ & 0.6782 & 0.6889 & 0.6897 & NA \\
GO & 0.8321 & 0.8268 & 0.7981 & $\mathbf{0 . 8 5 8 3}$ & NA \\
GTEx & $\mathbf{0 . 8 1 6 3}$ & 0.7114 & 0.7233 & 0.7476 & NA \\
pathDIP & $\mathbf{0 . 8 4 2 0}$ & 0.8314 & 0.7607 & 0.8051 & NA \\
Mashup & $\mathbf{0 . 8 6 3 1}$ & NA & NA & NA & NA \\
KEGG & $\mathbf{0 . 8 3 2 4}$ & NA & NA & NA & NA \\
Concat. M & 0.8881 & 0.8688 & 0.8760 & NA & $\mathbf{0 . 9 1 5 3}$ \\
Concat. P\&G & 0.7995 & 0.8503 & 0.8777 & $\mathbf{0 . 8 7 9 5}$ & $\mathbf{0 . 9 0 9 5}$ \\
\hline
\end{tabular}


positive rate (FPR), and the ordinate is the true positive rate (TPR). Their calculation formulas are as Eq. (4):

$$
\mathrm{FPR}=\frac{F P}{F P+T N}, \mathrm{TPR}=\frac{T P}{T P+F N} .
$$

where FN, FP, TN, TP mean false negative, false positive, true negative and true positive, respectively. Because some genes have unknown values of features for each type of feature, we use two different strategies to generate the training and test sets according to whether or not genes with unknown features are all included in those datasets. Tables 3 and 4 show the value of the AUROC measure obtained by each method, for the datasets containing or not (respectively) the genes with unknown values of the corresponding type of feature. The bold in tables 3 and 4 represents the prediction results of the best algorithm in the corresponding dataset.

It should be noted that the data in the first four rows (PPI, GO, GTEx, PathDIP) of columns 3-5 (GBT, LR, DNN) in Tables 3 and 4 are provided by Fabris et al. [7]. From the data in the above two tables, it can be seen that the effect of only containing known genes is better than that of containing unknown genes. In Table 3, when using only the PPI data set, our Encoder 1 is $5.44 \%$ lower than Navie, but it is more than $6 \%$ higher than other algorithms, and more than $10 \%$ higher than the previous DNN algorithm. When using the GO data set alone, Encoder1 is slightly lower than DNN. When using other data sets, our algorithm will perform better than other algorithms.

In Table 4, we use Mashup algorithm combined with the MDL model, which has more advantages than the existing deep learning algorithm or the traditional machine learning algorithms such as logistic regression algorithm and gradient boosting tree. When we directly use PPI or GO data to input Encoder1, too large data dimensionality leads to a huge increase in the amount of calculation, and the convergence speed is also very slow (10-fold cross-validation training time is about $10 \mathrm{~h}$ per module). When we use the Mashup algorithm to combine PPI and GO, it not only greatly reduces the amount of machine learning computation, but also improves the prediction effect. From 0.7464 of PPI and 0.8321 of GO to 0.8631 of Mashup. Then we add all the data sets into Encoder2, that is, we bring them into Concat. P\&G respectively to make the final prediction. Although our data sample dimensions are larger $(16 * 5$ vs $16 * 4$, where 16 is the each feature data dimensions after Dimensionality reduction by Encoder1), we still have advantages in the prediction effect $(0.9095$ vs 0.8795$)$. It proves that our deep learning model has better effect than the previous deep learning model. Finally, by comparing the training effect of encoder2 using concat. $M$ and concat. $P \& G$ data sets, we find that the use of concat. $\mathrm{M}$ also has a significant improvement ( 0.9153 vs 0.9095$)$. This shows that mashup algorithm not only has a good effect in embedding PPI and GO, but also has a positive effect in integrating it into other feature data sets.

In order to see the advantages of our method more intuitively, We draw ROCs in each method in the case of using Concat. M or Concat. P\&G datasets. It is worth noting that the larger the area under the curve, that is, the higher the curve is, the better the prediction effect of the corresponding algorithm.

The corresponding results of Fig. 2 are the rows concat. $M$ and concat. P\&G in Table 4. GBT, GBT0 use GBT algorithm, LR, LR0 use LR algorithm, fulldataset and fulldataset0 


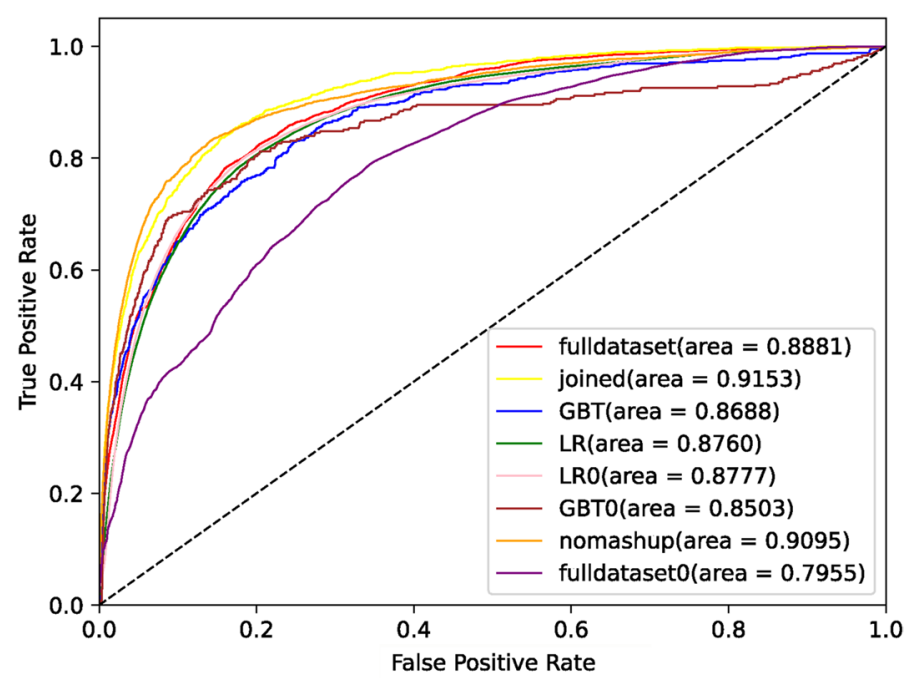

Fig. 2 Comparison of ROCs by Concat. M datasets and Concat. P\&G datasets on each methods

only use Encoder1 algorithm, the data set Concat. $M$ is used if the suffix of their names contains 0 , otherwise Concat. $P \& G$ is used. Joined and nomashup correspond to the two results of whether our MDL algorithm uses Mashup for embedding in the dataset PPI and GO. In Fig. 2, we can see that 'joined' is significantly higher than all other methods except 'nomashup'. We can also see that directly concatenating several large data sets together using Encoder1 will also seriously reduce the training effect.

\section{Results statistical analysis}

We use our own method to compare existing DNN algorithms, GBT and logistic regression classification, and make a statistical analysis in the case of considering the use of known and unknown genes. Considering that the data set used in a single module is different from that used in the combination of all our data, and the prediction performance is obviously better in the case of combining multiple data sets, we do not give statistical analysis of the results for the individual datasets (types of features), and focus instead on the statistical analysis for the results combining all datasets. Our statistical analysis method includes two kinds, one is based on T-test, which is used to test whether there is significant difference in the mean value of cross validation results. We add the statistical significance test based on Mann-Whitney-U test to supplement. In the U-test, we calculate the $p$ value of "Greater", "Two side" and "Less" models, that is, the null hypothesis is that other methods are better, the same or worse than ours. The data we tested and analyzed were 10 -folds cross validation of 30 random seed changes, and the AUC value predicted by each fold record was verified.

The data in Table 5 shows the comparison results between our method and other methods. The first column lists our method and each method that needs to be compared. The second column is the combination method of each characteristic data set under different methods. The third column is the corresponding average AUROC value. The fourth column gives the significance test results of $T$ hypothesis. The fifth column 
Table 5 Comparing the MDL approach $(A U R O C=0.9153)$ with each data set of others methods using T-test and Mann-Whitney-U hypothesis testing

\begin{tabular}{lllllll}
\hline Algorithms & Feature combination & AUROC & T-test & & \multicolumn{3}{l}{ Mann-Whitney-U test $p$ value } \\
\cline { 6 - 7 } & & & $p$ value & Greater & Two-side & Less \\
\hline Encoder1 & Concat. M & 0.8881 & 0.0045 & 0.0021 & 0.0043 & 0.9980 \\
& Concat. P\&G & 0.7955 & $1.721 \mathrm{e}-09$ & $4.428 \mathrm{e}-08$ & $8.857 \mathrm{e}-08$ & 1.0000 \\
Encoder2 & Concat. P\&G & 0.9095 & 0.1256 & 0.0261 & 0.0522 & 0.9739 \\
GBT & Concat. M & 0.7301 & $6.778 \mathrm{e}-09$ & $4.523 \mathrm{e}-08$ & $9.046 \mathrm{e}-08$ & 1.0000 \\
& Concat. P\&G & 0.8503 & 0.0001 & $4.146 \mathrm{e}-05$ & $8.292 \mathrm{e}-05$ & 1.0000 \\
LR & Concat. M & 0.8760 & $1.704 \mathrm{e}-06$ & $1.461 \mathrm{e}-07$ & $2.922 \mathrm{e}-07$ & 1.0000 \\
& Concat. P\&G & 0.8777 & $4.071 \mathrm{e}-07$ & $1.094 \mathrm{e}-07$ & $2.189 \mathrm{e}-07$ & 1.0000 \\
\hline
\end{tabular}

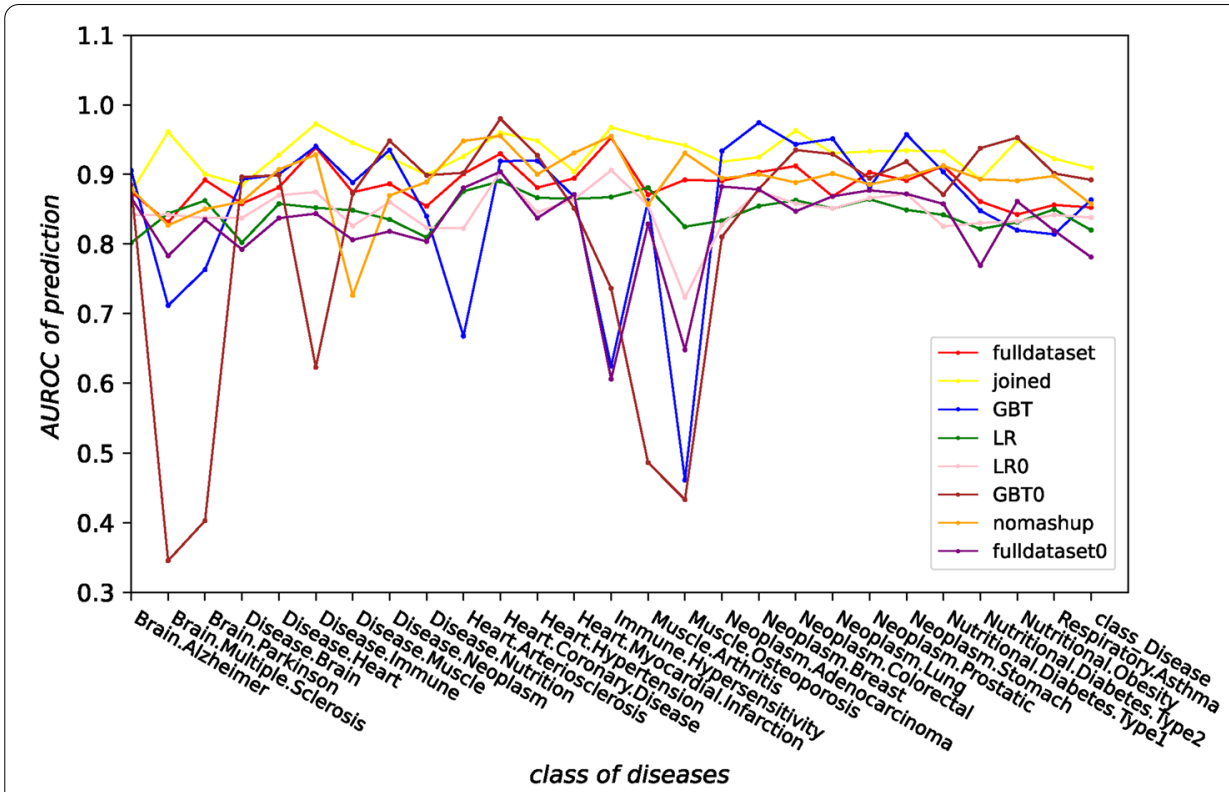

Fig. 3 AUROC value of 27 diseases predicted by each algorithm

is the statistical results based on Mann-Whitney-U test. From this table, we can see that except for the prediction results in the line of Encoder2, other methods can easily reject the null hypothesis through $p$ value and get the conclusion that our method works better. However, using Encoder2 to combine data with Concat. P\&G method can not reject the null hypothesis of T-test $(0.1256<0.05)$, and their average value has no significant difference. At the same time, in Mann-Whitney-U test, the p value of Encoder2 algorithm in Concat. P\&G is better than Concat. $M$ is 0.0261 , which can reject the null hypothesis. Therefore, it can be said that at least we embed PPI and GO datasets in the way of Mashup, and then use our deep learning algorithm will not be worse than using PPI and GO datasets directly. 


\section{AUROC results of individual diseases}

In the end, we calculate by prediction AUROC of each class label (disease) for the final results predicted by all methods, and the AUROC is shown in Fig. 3. As we can see from Fig. 3 that the stability of prediction AUROC for each disease is the best among all methods, and our algorithm has the best performance in most diseases.

\section{Analysis of prediction results}

In the last step of prediction, we rank each disease score corrsponding to each gene in order to identify the positive (with disease) class labels recommended by the model. The set of samples (genes) predicted as having a positive class label by the model are likely to contain some negative samples (i.e. the corresponding diseases are not annotated), and these negative samples are the objects that we need to further study [22, 23]. Because the genes without annotation information are regarded as negative labeled samples by default, the originally positive samples may be wrongly annotated as negative in the dataset. It seems that the reliability of negative label is low, and the lack of relevant evidence does not mean that there is no evidence of disease [24].

In order to identify these negative genes more reliably, we carried out the following rule screening. We ranked all the negative genes according to the predicted probability that the gene is associated with each disease (i.e. the predicted probability of each positive class label), and selected the top 30 genes with negative labels with the highest probability, since the disease (class label) with less annotated genes, osteoporosis, contains only 30 genes, then we chose to recommend 30 genes for each disease. Since there is generally around 15,000 genes in the sample, which means at least the top $0.2 \%$, i.e. $30 / 15,000$, of the predicted genes met the requirements. Because of the randomness of deep learning, we repeated this experiment for 30 times, and required at least 20 times to meet the above requirements before we could get our recommendation for negative label samples. In fact, all the 30 recommended genes met this preset standards, furthermore, among the top 30 genes there are at least 25 genes were listed in the practical process of 30 repeats, exceeding the above standards.

Due to the limitation of space, instead of listing all the 30 positive genes, We only listed the top five genes in three of the 27 diseases. The 27 kinds of labels (diseases) include one primary label(Class_Disease), seven secondary labels(Disease.Brain, Disease.Heart, Disease.Immune, Disease.Muscle, Disease.Neoplasm, Disease.Nutrition, Disease.Respiratory.Asthma), and the remaining 19 are tertiary labels. The secondary label is defined as: if a gene associated with any one of the tertiary labels (disease) of the corresponding class, the secondary label is associated with the gene. Primary label is the same. We have chosen three of the seven more comprehensive secondary labels (Disease.Immune, Disease.Brain, Disease.Nutritional). Giving their average probability (Ave. P), the lowest probability (Low. P) and the highest probability (Hig. P) of positive prediction according to the frequency of occurrence in 30 repeated experiments. These results are summarized in Table 6, in which column 1 is the class of disease, column 2 is the Entrez ID of the gene and the official abbreviation of the name and the official full name. column $3-5$ is the average, highest, lowest probability of recommended gene in 30 experiments. Column 6 is the average probability of all negative genes except as recommended (top 
Table 6 The top five genes related to each of the three diseases according to the frequency of occurrence in 30 repeated experiments, and the various probabilities of association with disease of these candidate genes

\begin{tabular}{|c|c|c|c|c|c|}
\hline Disease & Candidate genes & Ave. $\mathrm{P}$ & Hig. P & Low. $\mathrm{P}$ & Ave. $P$ of rest genes \\
\hline \multirow[t]{7}{*}{ Immune } & 3553(IL1B -interleukin 1 beta) & 0.119864 & 0.166426 & 0.062936 & \multirow[t]{7}{*}{0.00128} \\
\hline & 1544(CYP1A2 — cytochrome P450 & 0.115728 & 0.159259 & 0.061422 & \\
\hline & family 1 subfamily A member 2) & & & & \\
\hline & 4023(LPL_ lipoprotein lipase) & 0.113289 & 0.155988 & 0.06022 & \\
\hline & 1543(CYP1A1 - cytochrome P450 & 0.111613 & 0.154343 & 0.059018 & \\
\hline & family 1 subfamily A member 1 ) & & & & \\
\hline & 4846(NOS3_nitric oxide synthase 3) & 0.110097 & 0.154304 & 0.057448 & \\
\hline \multirow[t]{9}{*}{ Brain } & 1544(CYP1A2 — cytochrome P450 & 0.204762 & 0.303633 & 0.111395 & \multirow[t]{9}{*}{0.0363} \\
\hline & family 1 subfamily A member 2) & & & & \\
\hline & 1559(CYP2C9_cytochrome P450 & 0.190703 & 0.28241 & 0.104468 & \\
\hline & family 2 subfamily C member 9 ) & & & & \\
\hline & 1586(CYP17A1 — cytochrome P450 & 0.175779 & 0.243321 & 0.104167 & \\
\hline & family 17 subfamily A member 1) & & & & \\
\hline & 338(APOB_apolipoprotein B) & 0.174123 & 0.256506 & 0.097296 & \\
\hline & 1557(CYP2C19-cytochrome P450 & 0.173544 & 0.258927 & 0.0953 & \\
\hline & family 2 subfamily ( member 19 ) & & & & \\
\hline \multirow[t]{8}{*}{ Nutrition } & 3553(IL1B -interleukin 1 beta) & 0.343483 & 0.496965 & 0.195028 & \multirow[t]{8}{*}{0.00677} \\
\hline & 1544(CYP1A2_cytochrome P450 & 0.332656 & 0.478875 & 0.190983 & \\
\hline & family 1 subfamily A member 2) & & & & \\
\hline & 1557(CYP2C19-cytochrome P450 & 0.284008 & 0.414314 & 0.163521 & \\
\hline & family 2 subfamily $($ member 19$)$ & & & & \\
\hline & 4035(LRP1_LDL receptor) & 0.280427 & 0.412398 & 0.159798 & \\
\hline & related protein 1 & & & & \\
\hline & 2688(GH1_growth hormone 1) & 0.279018 & 0.381114 & 0.171254 & \\
\hline
\end{tabular}

30) genes. As can be seen from Table 6, the probability that the genes we selected will be positive according to the algorithm is far greater than other genes.

Considering that the significance of comparing the best 30 genes recommended by the algorithm with the average value of all other negative genes may not be comprehensive enough (other genes may contain a lot of samples with low recommendation values, thus pulling down some samples similar to the recommended genes). So we used the same method of selecting recommended genes to select genes with probabilities ranging from 31 to 60 for each disease. And then we use the probability of their association with the disease and the top 30 genes for hypothesis testing. The null hypothesis is that the gene we recommend is the same as his neighbor.

It can be seen from Table 7 that the probability of our top 30 genes is significantly better than that of our neighbor genes (ranking 31-60). It should be noted that the statistics can't show that the negative class-label genes ranked after the 30th have no relationship with the diseases we study. On the contrary, if the genes ranked after the 30th have evidence related to a certain disease, according to our algorithm, the top 30 genes recommended by us should also have a higher probability of obtaining evidence related to diseases. 
Table 7 Testing results for the statistical hypothesis of whether the recommended 30 genes are significantly different from the subsequent 30 genes for 27 aging-related diseases

\begin{tabular}{llll}
\hline Disease classification & $p$ values & Disease classification & $\boldsymbol{p}$ values \\
\hline Disease.Brain & $8.484 \mathrm{e}-09$ & Disease.Neoplasm & $2.033 \mathrm{e}-09$ \\
Brain.Alzheimer & $3.474 \mathrm{e}-10$ & Neoplasm.Adenocarcinoma & $1.464 \mathrm{e}-10$ \\
Brain.Multiple.Sclerosis & $5.967 \mathrm{e}-09$ & Neoplasm.Breast & $1.205 \mathrm{e}-10$ \\
Brain.Parkinson & $1.070 \mathrm{e}-09$ & Neoplasm.Colorectal & $2.154 \mathrm{e}-10$ \\
Disease.Heart & $5.072 \mathrm{e}-09$ & Neoplasm.Lung & $7.380 \mathrm{e}-10$ \\
Heart.Arteriosclerosis & $1.776 \mathrm{e}-10$ & Neoplasm.Prostatic & $3.196 \mathrm{e}-09$ \\
Heart.Coronary.Disease & $6.695 \mathrm{e}-11$ & Neoplasm.Stomach & $2.609 \mathrm{e}-10$ \\
Heart.Hypertension & $4.504 \mathrm{e}-11$ & Disease.Nutrition & $7.118 \mathrm{e}-09$ \\
Heart.Coronary.Disease & $6.695 \mathrm{e}-11$ & Nutritional.Diabetes.Type1 & $3.689 \mathrm{e}-11$ \\
Disease.Immune & $6.065 \mathrm{e}-10$ & Nutritional.Diabetes.Type2 & $2.227 \mathrm{e}-09$ \\
Immune.Hypersensitivity & $5.494 \mathrm{e}-11$ & Nutritional.Obesity & $4.975 \mathrm{e}-11$ \\
Disease.Muscle & $2.438 \mathrm{e}-11$ & Disease.Respiratory.Asthma & $7.380 \mathrm{e}-10$ \\
Muscle.Arthritis & $1.464 \mathrm{e}-10$ & Class_Disease & $2.831 \mathrm{e}-08$ \\
Muscle.Osteoporosis & $8.484 \mathrm{e}-09$ & & \\
\hline
\end{tabular}

\section{Discussion}

In fact, the evidence of genes and related diseases that we recommend can also be found in a large number of literatures. For example, $I L 1 B$ of immune related diseases was approved by Zhi-gang et al. [25]. Found that it is related to immune T cells in abortion of pregnant women, and found in the paper 2016 that the lineal homologous gene of $I L 1 B$ also plays an important role in the immune regulation of fish [26]. Liver IL1B may be a new target for restoring bile and sterol transport to treat PNAC [27, 28]. The specific expression of CYP2C9 in the axons and synapses of brain neurons may be related to some brain diseases, and the mutation allele frequency of $C Y P 2 C 9$ is higher in 23 tumor samples, such as breast tumor and brain tumor, which indicates that CYP2C9 may be related to tumor $[29,30]$.

The evidence we found is not limited to these genes. With the help of Google academic, we can easily find the research papers related to the recommended genes for each disease. For the 15 genes mentioned above, we list the relevant information in the Table 8. Finally, only the evidence of CYP2C19 gene recommended for nutritional diseases was not found.

We have recommended 30 genes that are most likely to be positive for each disease. However, due to the limitation of space, we have only made a detailed analysis of five genes of three diseases (Disease.Immune, Disease.Brain, Disease.Nutritional). But we want to find out whether there is some internal relationship between these genes recommended for each disease, so as to find out the genes that may be related to aging. Therefore, we have made the Fig. 4 for all the recommended genes, so we can see their distribution more intuitively. Figure 4 should be viewed horizontally.

The Vertical-axis of the five subgraphs in Fig. 4 corresponds to 27 diseases. The order of the Vertical-axis from 1 to 27 corresponds to the order from top to bottom listed on the right of the figure. The Horizontal-axis of each subgraph corresponds to the ID value of the recommended gene. 
Table 8 Evidence of 15 genes recommended for association with disease

\begin{tabular}{|c|c|c|}
\hline Gene ID & Genes associated with “Disease.Immune” & Relevant evidence \\
\hline 3553 & IL $1 B$-interleukin 1 beta & Roghieh Safari et al. [26] \\
\hline \multirow[t]{2}{*}{1544} & CYP1A2_cytochrome P450 family 1 & Klein et al. [31] \\
\hline & subfamily A member & \\
\hline 4023 & LPL_lipoprotein lipase & S. aureus et al. 2015 [32] \\
\hline \multirow[t]{2}{*}{1543} & CYP1A1_cytochrome P450 family 1 & Uno et al. [33] \\
\hline & subfamily A member 1 & \\
\hline 4846 & NOS3 — nitric oxide synthase 3 & Bogdan et al. [34] \\
\hline Gene ID & Genes associated with "Disease.Brain" & Relevant evidence \\
\hline \multirow[t]{2}{*}{1544} & CYP1A2_cytochrome P450 family 1 & Siokas et al. [35] \\
\hline & subfamily A member 2 & \\
\hline \multirow[t]{2}{*}{1559} & CYP2C9_cytochrome P450 family 2 & Sun et al. [36] \\
\hline & subfamily C member 9 & \\
\hline \multirow[t]{2}{*}{1586} & CYP17A1 — cytochrome P450 family 17 & Emanuelsson et al. [37] \\
\hline & subfamily A member 1 & \\
\hline 338 & $A P O B$-apolipoprotein B & Bjelik et al. [38] \\
\hline \multirow[t]{2}{*}{1557} & CYP2C19-cytochrome P450 family 2 & Ingelman-Sundberg et al. [39] \\
\hline & subfamily C member 19 & \\
\hline Gene ID & Genes associated with "Disease.Nutrition" & Relevant evidence \\
\hline 3553 & IL1B-interleukin 1 beta & Norde et al. [40] \\
\hline \multirow[t]{2}{*}{1544} & CYP1A2_cytochrome P450 family 1 & Agúndez et al. [41] \\
\hline & subfamily A member 2 & \\
\hline \multirow[t]{2}{*}{1557} & CYP2C19-cytochrome P450 family 2 & None \\
\hline & subfamily C member 19 & \\
\hline 4035 & LRP1_LDL receptor related protein 1 & Masson et al. [42] \\
\hline 2688 & GH1_growth hormone 1 & Thissen et al. [43] \\
\hline
\end{tabular}

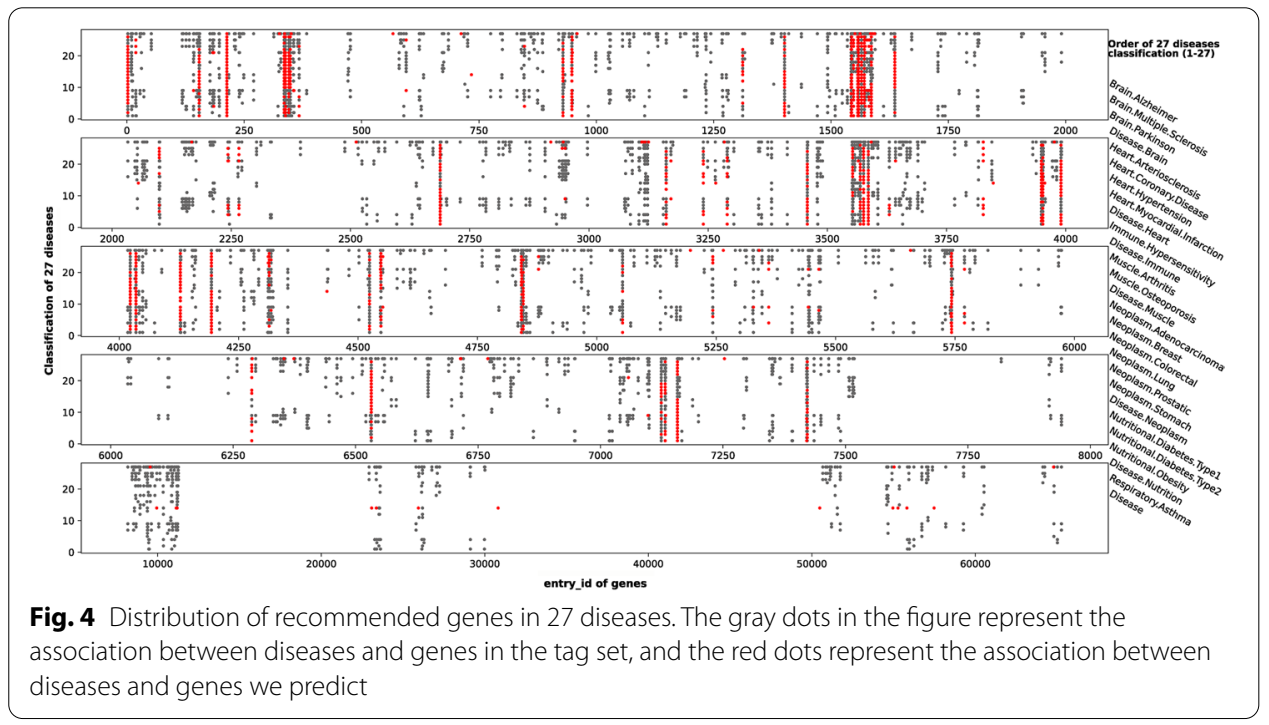

As can be seen from the Fig. 4, the majority of the recommended genes are distributed within 1-8000, and after 8000 only the category of diseases exists with recommended genes. The distribution of these recommended genes in 1-8000 shows that many of our 
recommended genes like to cluster in multiple diseases. Although we have not made further analysis on each type of disease, it can be seen from the gene prediction results that it is more likely that similar diseases will predict the same associated genes. This phenomenon shows that the diseases we choose have certain internal relations, and as far as we know, the only thing they have in common may be that they are all related to aging. This also shows that our research can not only stay in the level related to aging diseases, but also have a certain reference value in the deeper level of the relationship between genes and aging. Some recommended genes appear frequently in some regions, such as $300-400,1500-1600,3500-3600,7100-7200$, and so on. It may be more helpful to reveal the relationship between genes and aging by giving priority to gene research in these regions. An additional file shows all recommended genes in more detail (see Additional file 1).

\section{Conclusion}

In general, our method has a significant improvement in prediction performance compared with the existing modular deep learning model or the traditional machine learning method like LR or GBT in both single module prediction and total module prediction accuracy. At the same time, for a single module, we use Mashup algorithm to embed PPI and GO into a network, which greatly improves the original performance of only two modules. In addition, due to the decline of data dimensionality, the training time also has a great decline (from dozens of hours to only more than $1 \mathrm{~h}$ ). We used 30 times of random seed replacement to repeat the experiment. The 30 negative marker genes were recommended for further analysis by biological researchers with more than $99.8 \%$ (i.e. 1 - $(30 / 19,188))$ positive probability. The hypothesis test of the top 30 genes in our experiment and the genes ranking 31-60 confirmed that the recommended genes are significantly different from other genes. We also found evidence from the literature that supports our assumption that genes may be disease-related, which further confirms that lack of evidence of gene-disease association does not mean they are not disease related.

Abbreviations

MDL: Mashup and deep learning; PPI: Protein-protein interactions; GO: Gene ontology; KEGG: Kyoto Encyclopedia of genes and genomes Genes; GTEx: The genotype-tissue expression (GTEx) project.

\section{Supplementary Information}

The online version contains supplementary material available at https://doi.org/10.1186/s12859-021-04518-5.

Additional file 1: Details of predicted genes.

\section{Acknowledgements}

We thank anonymous reviewers for valuable suggestions.

Authors' contributions

$J Y$ and SW designed the research and wrote the manuscript; JY performed the experiments and analyses; $X Y$ and

XT assisted in data preparation and code development. SW and XT performed English editing. All authors read and approved the manuscript.

\section{Funding}

This research is fully supported by grants from National Natural Science Foundation of China (62062067), Natural Science Foundation of Yunnan Province (2017FA032) and Training Plan for Young and Middle-aged Academic Leaders of Yunnan Province (2018HB031). 
Availability of data and materials

The source code and datasets located https://github.com/jhuaye/GenesRelateDiseases.

\section{Declaration}

Ethics approval and consent to participate

Not applicable.

\section{Consent for publication}

Not applicable.

\section{Competing interests}

The authors declare that they have no competing interests.

Received: 6 June 2021 Accepted: 30 November 2021

Published online: 17 December 2021

\section{References}

1. Indika K, Christopher F, Karin V, Asa B-H. Phenostruct: prediction of human phenotype ontology terms using heterogeneous data sources. F1000Research. 2015;4:259-259.

2. Angermueller C, PaRnamaa T, Parts L, Stegle O. Deep learning for computational biology. Mol Syst Biol. 2016;12(7):878.

3. Fernandes M, Cen W, Tacutu R, Barardo D, Magalhes JPD. Systematic analysis of the gerontome reveals links between aging and age-related diseases. Hum Mol Genet. 2016;25(21):307.

4. Shi K, Gao L, Wang B. Systematic tracking of coordinated differential network motifs identifies novel disease-related genes by integrating multiple data. Neurocomputing. 2016;206:3-12 (SI:DMSB).

5. Lu C, Yang M, Luo F, Fang-Xiang W, Li M, Pan Y, Li Y, Wang J. Prediction of Incrna-disease associations based on inductive matrix completion. Bioinformatics. 2018;19:3357-64.

6. Cho H, Berger B, Peng J. Compact integration of multi-network topology for functional analysis of genes. Cell Syst. 2016;3:540.

7. Fabio F, Daniel P, Salama KM, Pedro D, Freitas AA. Using deep learning to associate human genes with age-related diseases. Bioinformatics. 2019:7:7.

8. Meng Q. Lightgbm: a highly efficient gradient boosting decision tree. 2018.

9. Shevade SK, Keerthi SS. A simple and efficient algorithm for gene selection using sparse logistic regression. Bioinformatics. 2003;19(17):2246-53.

10. Zeng X, Liao Y, Liu Y, Zou Q. Prediction and validation of disease genes using hetesim scores. IEEE/ACM Trans Comput Biol Bioinf. 2017;14(3):687-95. https://doi.org/10.1109/TCBB.2016.2520947.

11. Fabio F, Freitas AA. New KEGG pathway-based interpretable features for classifying ageing-related mouse proteins. Bioinformatics. 2016:32(19):363.

12. Damian S, Morris JH, Helen C, Michael K, Stefan W, Milan S, Alberto S, Doncheva NT, Alexander R, Peer B. The STRING database in 2017: quality-controlled protein-protein association networks, made broadly accessible. Nucleic Acids Res. 2017;45:362-8.

13. Ashburner M, Ball CA, Blake JA, Botstein D, Butler H, Cherry JM, Davis AP, Dolinski K, Dwight SS, Eppig JT. Gene ontology: tool for the unification of biology. Nat Genet. 2000;25:25-9.

14. Alshahrani M, Hoehndorf R. Semantic disease gene embeddings (SmuDGE): phenotype-based disease gene prioritization without phenotypes. Bioinformatics. 2018;34:i901-7.

15. Wang S, Cao Z, Li M, Yue Y. G-DipC: an improved feature representation method for short sequences to predict the type of cargo in cell-penetrating peptides. IEEE/ACM Trans Comput Biol Bioinform. 2019;17:739-47.

16. Wang S, Li M, Guo L, Cao Z, Fei Y. Efficient utilization on PSSM combining with recurrent neural network for membrane protein types prediction. Comput Biol Chem. 2019;81:9-15.

17. Daniel Q, Xie X. DANQ: a hybrid convolutional and recurrent deep neural network for quantifying the function of DNA sequences. Nucleic Acids Res. 2016;11:107-107.

18. Kulmanov M, Khan MA, Hoehndorf R. DeepGO: predicting protein functions from sequence and interactions using a deep ontology-aware classifier. Bioinformatics. 2017:34(4):660-8.

19. Skwark MJ, Raimondi D, Michel M, Elofsson A. Improved contact predictions using the recognition of protein like contact patterns. PLoS Comput Biol. 2014;10(11):1003889.

20. Gupta A, Wang H, Ganapathiraju M. Learning structure in gene expression data using deep architectures, with an application to gene clustering. In: IEEE international conference on bioinformatics and biomedicine. 2015.

21. Manessi F, Rozza A. Learning combinations of activation functions. In: 2018 24th international conference on pattern recognition (ICPR). 2018

22. Niehaus K. Learning with noisy labels. 2014

23. Yu HF, Jain P, Kar P, Dhillon IS. Large-scale multi-label learning with missing labels. 2013.

24. Gillis J, Pavlidis P. The impact of multifunctional genes on "guilt by association" analysis. PLoS ONE. 2011;6(2):17258.

25. Wang Z, Hill J, Yunis E, Xiao L, Anderson D. Maternal cd46h*2 and il 1 b- $511^{*} 1$ homozygosity in t helper 1-type immunity to trophoblast antigens in recurrent pregnancy loss. Hum Reprod. 2006;21:818-22. https://doi.org/10.1093/ humrep/dei366.

26. Safari R, Hoseinifar SH, Nejadmoghadam S, Jafar A. Transciptomic study of mucosal immune, antioxidant and growth related genes and non-specific immune response of common carp (Cyprinus carpio) fed dietary ferula (Ferula assafoetida). Fish Shellfish Immunol. 2016:55:242-8. 
27. Stefanidis I, Kreuer K, Dardiotis E, Arampatzis S, Eleftheriadis T, Hadjigeorgiou GM, Zintzaras E, Mertens PR. Association between the interleukin-1 $\beta$ gene (il1 b) c-511t polymorphism and the risk of diabetic nephropathy in type 2 diabetes: a candidate-gene association study. DNA Cell Biol. 2014;33(7):463-8.

28. Kasmi KE, Vue PM, Anderson AL, Devereaux MW, Ghosh S, Balasubramaniyan N, Fillon SA, Dahrenmoeller C, Allawzi A, Woods C. Macrophage-derived il-1 $\beta / \mathrm{nf}-\mathrm{kb}$ signaling mediates parenteral nutrition-associated cholestasis. Nat Commun. 2018;9(1):1393.

29. Knüpfer H, Stanitz D, Preiss R. Cyp2c9 polymorphisms in human tumors. Anticancer Res. 2006;26(1A):299-305.

30. Depaz I, Toselli F, Wilce PA, Gillam EM. Differential expression of cytochrome p450 enzymes from the cyp2c subfamily in the human brain. Drug Metab Dispos. 2015;43(3):353-7.

31. Kathrin K, Stefan W, Miia T, Matthias S, Zanger UM. Pathway-targeted pharmacogenomics of cypla2 in human liver. Front Pharmacol. 2010;1(129):129.

32. Minh T, Nguyen B, Kraft W, Yu D, Doruk D. The u saa specific lipoprotein like cluster (Ipl) of S. aureus USA300 contributes to immune stimulation and invasion in human cells. Plos Pathog. 2015;11:e1004984.

33. Uno S, Dalton TP, Dragin N, Curran CP, Nebert DW. Oral benzo[a]pyrene in cyp1 knockout mouse lines: Cyp1a1 important in detoxication, cyp 1 b1 metabolism required for immune damage independent of total-body burden and clearance rate. Mol Pharmacol. 2006;69(4):1103-14.

34. Bogdan C. Nitric oxide and the immune response. Nat Immunol. 2001;2(10):907-16.

35. Siokas V, Karampinis E, Aloizou AM, Mentis A, Dardiotis E. Cyp1a2 rs762551 polymorphism and risk for amyotrophic lateral sclerosis. Neurol Sci. 2020;42(1):175-82.

36. Sun Y, Yu J, Yuan Q, Wu X, Wu X, Hu J. Early post-traumatic seizures are associated with valproic acid plasma concentrations and ugt1a6/cyp2c9 genetic polymorphisms in patients with severe traumatic brain injury. Scand J Trauma Resusc Emerg Med. 2017;25(1):85.

37. Emanuelsson I, Almokhtar M, Wikvall K, GrNbladh A, Nylander E, Svensson AL, Svenningsen F, Norlin M. Expression and regulation of cyp $17 \mathrm{a} 1$ and $3 \beta$-hydroxysteroiddehydrogenase in cells of the nervous system: potential effects of vitamin d on brain steroidogenesis. Neurochem Int. 2018;113:46-55.

38. Bjelik A, Bereczki E, Gonda S, Juhász A, Kálmán J. Human apoB overexpression and a high-cholesterol diet differently modify the brain APP metabolism in the transgenic mouse model of atherosclerosis. Neurochem Int. 2006;49(4):393-400

39. Ingelman-Sundberg M, Persson A, Jukic MM. Polymorphic expression of cyp2c19 and cyp2d6 in the developing and adult human brain causing variability in cognition, risk for depression and suicide: the search for the endogenous substrates. Pharmacogenomics. 2014;15(15):1841-4.

40. Norde MM, Oki E, Carioca A, Damasceno N, Rogero MM. Influence of IL1B, IL6 and IL10 gene variants and plasma fatty acid interaction on metabolic syndrome risk in a cross-sectional population-based study. Clin Nutr. 2018;37(2):659-66.

41. Agúndez J, Gallardo L, Martínez C, Gervasini G, Benítez J. Modulation of CYP1A2 enzyme activity by indoleamines: inhibition by serotonin and tryptamine. Pharmacogenetics. 1998;8(3):251-8.

42. Olivier M, Carine C, Cédric D, Aline M, Danielle D, Didier Q, Catherine M, Philippe V, Emmanuelle LC, Cao Y. LRP1 receptor controls adipogenesis and is up-regulated in human and mouse obese adipose tissue. PLOS ONE. 2009;4(10):7422.

43. Thissen JP. Nutritional regulation of the insulin-like growth factors. Endocr Rev. 1994;15(1):80-101.

\section{Publisher's Note}

Springer Nature remains neutral with regard to jurisdictional claims in published maps and institutional affiliations.

Ready to submit your research? Choose BMC and benefit from:

- fast, convenient online submission

- thorough peer review by experienced researchers in your field

- rapid publication on acceptance

- support for research data, including large and complex data types

- gold Open Access which fosters wider collaboration and increased citations

- maximum visibility for your research: over $100 \mathrm{M}$ website views per year

At BMC, research is always in progress.

Learn more biomedcentral.com/submissions 